\title{
Editorial: Health informatics
}

\author{
R. J. McClelland
}

"The importance of up to date information, and the key role played by the medical profession in supplying the valid data from which it is derived, is not yet understood by many senior medical staff, let alone those in training." Williams et al, 1992.

\section{The need for information}

In many important respects the nature of professional practice in psychiatry and in each of its specialities remains fundamentally unchanged across one's professional lifetime. Nevertheless, the context in which we carry out our task has changed dramatically and there is little reason to suppose that changes will not continue to happen. Not all change is necessarily bad and some may even provide new incentives and new means to improvements in clinical practice and in the quality of services received by patients and their carers.

One important cultural change throughout health services is a greater emphasis on the consumer and the service user perspective. With this has come growing demands for greater professional accountability and a growing expectation of patient participation in clinical decisionmaking. These changes impinging on medical practice from the outside have their counterparts from within the profession. They can be seen throughout the spectrum of medical education from undergraduate to postgraduate and continuing professional development (CPD). One example is the new emphasis placed on a critical approach to evidence, a fundamental principle in the General Medical Council guidelines (1993) for undergraduate medical education, 'Tomorrow's Doctors'. Another is the new Research and Development Strategy within the NHS stressing the importance of evidence-based medicine and medical practice.

All these changes from without and within, place a great expectation on availability of appropriate, timely and accurate information for professionals, for service users, for carers, for commissioners of services and for policy-makers. The information required is more than the rarefied evidence from clinical research and experimental medicine under the refined conditions of the experimental laboratory, be it at the bench or in the community. The information necessary relates to routine clinical practice in all its aspects from doctor/ patient communication through prescribing of medical treatments to social interventions. It includes information pertaining to the process of care, the outcome of care, resource usage and resource costs.

Health informatics is part of the science of informatics and refers to the processing of health information/data in all its aspects including: what information clinicians need; clinical data storage and retrieval; the analysis and interpretation of routine clinical information; and security and confidentiality of clinical information systems.

This issue of APT focuses on the information needs of psychiatrists and the opportunities afforded by current developments in health informatics and parallel developments in Community Information Systems. Four experts have been invited to address this vacuum in professional education. They bring four complementary perspectives - clinician, college, Department of Health, and the Management Executive's Information Management and Technology Strategy.

\section{Professional incentive}

From the perspective of adult education and professional development, there are two key questions. The first is professional incentive - why should busy clinicians take an interest in and learn about information management, information technology, information systems? One of the 
linchpins in this argument is the emerging question of clinical effectiveness - how good, how effective is the service we provide? Ten years ago the health economist Maxwell (1984) urged that quality and effectiveness of care should be at the centre of the health care debate, as they are in the end the most important dimensions of the health care performance. However, Maxwell also reminds us that an honest concern about quality is not methodological assessment based on reliable information, clinical measurement and monitoring. There is a real risk today that if we fail to grapple with this information and measurement issue in mental health services then costs and volumes which can readily be quantified will drive health services activity.

Professional attention has traditionally focused on the resource side of the Donabedian triad: resource, process, outcome (Donabedian, 1980). More recently, through medical and, to a lesser extent, clinical audit attention has begun to focus on the process side. There is a prima facie case for a new and complementary focus on the third pole of this triad - mental health outcomes. Considerable effort over many years of health service research has enabled us to identify new and efficacious treatments and service interventions which have transformed the quality of life for many of our patients. However, in the UK there is an urgent need to translate such efficacy research into clinical effectiveness through appropriate routine assessment of clinical outcomes. Such clinical effectiveness will form the bedrock of any resource management, cost-effectiveness strategy. Here the needs of professionals and management converge.

There is a clear and parallel message from the US. Stephen Sharstein, Professor of Psychiatry at the University of Maryland reports a profound reassessment in American healthcare services. He predicts a new focus on quality. What US citizens will pay for will increasingly be informed by what actually works, what is most effective - a shift from process to results and outcomes (Sharstein, 1993). The Government's 'Health of the Nation' strategy has made health gain for the mentally ill one of its priorities. We have, therefore, for the first time a formal public and political incentive for improving health care outcomes. Information on patient progress and its measurement is a fundamental prerequisite.

To recap, there are strong incentives, if not imperatives, that clinicians begin as a matter of some urgency to address the whole matter of information and measurement in mental health.

\section{A new education agenda}

This highlights the second key question in professional development - the needs of clinicians, and the education agenda itself. The rapidly changing culture in which health care is practised demands that the present education agenda, undergraduate, postgraduate and CPD, is broadened to embrace health informatics. As the Director of the NHS Information Directorate recently noted, all senior clinicians should be fully aware of what IT can offer (Rogers, personal communication). Rogers also stresses the importance of professional bodies in this process. The Committee on Medical Information Technology of the Royal College of Physicians also stresses the need for educational initiatives to change the culture - "shaping attitudes so that quality information and communication are second nature; developing skills and knowledge so that data are collected and analysed with ease and valid, relevant and timely information is appraised and used with sense" (Williams et al, 1992). The report of the Royal College of Psychiatrists Mental Health Information Systems Working Group (1992) recommends that the College takes a lead in addressing the lack of knowledge and skills in the neglected area of mental health informatics. Many of the major issues in mental health informatics are addressed in this special issue of $A P T$.

\section{References}

Donabedian, A. C. (1980) The Definition of Quality and Approaches to its Assessment. Ann Arbor: Michigan Health Administration Press.

General Medical Council (1993) Tomorrow's Doctors. London: GMC.

Maxwell, R. (1984) Quality assessment in health. British Medical Journal, 288, 1470-1472.

Royal College of Psychiatrists (1992) Report of the Mental Health Systems Working Group.

Sharstein, S. (1993) Quality improvement. American Journal of Psychiatry, 150, 1767-1768.

Williams, J. G., de Dombal, F. T., Knill-Jones, R., et al (1992) Collecting, communicating and using information: the educational issues. Journal of the Royal College of Physicians, 26, 385-387. 